J. Gynäkol. Endokrinol. CH $2020 \cdot 23: 72-75$

https://doi.org/10.1007/s41975-020-00146-z

C Der/die Autor(en) 2020

\section{Fallbericht}

Eine 49-jährige Patientin stellt sich in der gynäkologisch-endokrinologischen Sprechstunde vor. Sie berichtet über seit Langem bestehende, in letzter Zeit jedoch zunehmende Müdigkeit und Leistungsschwäche. Insbesondere klagt sie über Ein- und Durchschlafstörungen. Morgens habe sie z.T. Probleme, wach zu werden, und tagsüber habe sie oftmals Phasen mit ausgeprägter Müdigkeit. Darüber hinaus käme es rezidivierend $\mathrm{zu}$ Unterbauchschmerzen, die sie jeweils durch Einnahme von Schmerzmitteln zu lindern versuche. Ein Bezug zum Zyklusgeschehen kann im Zustand nach Hysterektomie nicht hergestellt werden. Vor einem Jahr erfolgte durch den behandelnden Orthopäden bei ausgeprägten Gelenkbeschwerden eine mehrmonatige Kortisonbehandlung. Ebenso hat eine Behandlung mit Opioiden stattgefunden. Eine deutliche Besserung habe sich nicht ergeben, sodass sowohl Kortison als auch die Opioide inzwischen abgesetzt wurden. Die Beschwerden dauern nach wie vor an.

Die weitere Behandlung erfolgte über den Hausarzt. Es wurde zur weiteren Abklärung der Gelenkbeschwerden eine Osteodensitometrie durchgeführt. Es zeigte sich eine Osteopenie. Weiterhin seien mehrfach Laboruntersuchungen durchgeführt worden, die jedoch kein eindeutiges Ergebnis gezeigt hätten, allerdings sei beim letzten Mal der Schilddrüsenwert nicht in Ordnung gewesen und der Kortisolspiegel im Speichel (vom Heilpraktiker bestimmt) sei erhöht. Der Hausarzt nehme ihre Beschwerden nicht wirklich ernst, halte sie für eine „Simulantin“ und wolle keine Hormonbehandlung bei ihr einleiten, daher habe sie sich ,auf eigene Faust “ in unserer Sprechstunde angemeldet, um durch unsere Spezialisten eine

\title{
Christoph Keck
}

amedes Holding GmbH, Hamburg, Deutschland

\section{Der rätselhafte Fall}

entsprechende Behandlung einleiten $\mathrm{zu}$ lassen.

Zur Sozialanamnese berichtet die Patientin: geschieden, keine Kinder. Die Ehe sei wegen einer Endometriose kinderlos geblieben. Aufgrund persistierender, therapieresistenter Beschwerden erfolgte vor 10 Jahren eine Hysterektomie. Auch danach habe sie zeitweise wieder Schmerzen gehabt, die dann jedoch mit der Trennung von ihrem Mann wieder an Intensität zugenommen hätten.

Früher sei sie als Personalreferentin tätig gewesen, wegen lang dauernder Krankschreibung habe sie jedoch ihren Arbeitsplatz vor 3 Jahren verloren. Seitdem sei sie arbeitssuchend. Im Rahmen der Trennung kurzzeitig psychotherapeutische Behandlung wegen depressiver Episode.

\section{Diagnostik}

Die von der Patientin geschilderte Symptomatik kann auf den ersten Blick dazu verleiten, die Beschwerden als perimenopausales Geschehen zu interpretieren bzw. die Müdigkeit und den Leistungsabfall als Folge einer vermeintlichen Schilddrüsenunterfunktion $\mathrm{zu}$ interpretieren. Der Unterbauchschmerz könnte auch im Zustand nach Hysterektomie noch als persistierende Endometrioseaktivität gedeutet werden.

Zur Abklärung wurde daher zunächst der basale Hormonstatus mit folgenden Ergebnissen ermittelt (•Tab. 1)

\section{Interpretation der Befunde}

Es findet sich bei normalem Estradiolwert erwartungsgemäss eine perimenopausale Konstellation der Gonadotropine. Darüber hinaus lassen sich Normalwerte für Kortisol, Testosteron und Prolaktin darstellen. Bei leicht er- höhtem TSH-Wert und normalen fT3sowie fT4-Werten liegt eine latente Hypothyreose vor, die als nicht behandlungsbedürftig eingestuft wird.

Damit lässt sich eine endokrinologische Ursache der von der Pat. angegebenen Beschwerden mit hoher Wahrscheinlichkeit ausschliessen.

\section{Weiteres Vorgehen}

Die diffuse Konstellation der Beschwerden und der zeitliche Verlauf der geschilderten Symptomatik deuten darauf hin, dass möglicherweise keine rein somatische Erkrankung, sondern vielmehr ein psychisch bzw. psychosomatisch bedingtes Krankheitsgeschehen vorliegt. Es wird daraufhin eine weiterführende Untersuchung in der psychosomatischen Abteilung veranlasst.

Daraus ergeben sich die folgenden Diagnosen:

- Rezidivierende depressive Störung; gegenwärtig Remission (ICD-10: F33.1)

- Chronisches Schmerzsyndrom bei Fibromyalgie (ICD-10: M79.0)

Der Patientin wird daraufhin ein multimodales Behandlungskonzept bei Fibromyalgiesyndrom empfohlen.

Weshalb ist dieser Fallbericht überhaupt für Frauenärztinnen/Frauenärzte relevant?

1. In Deutschland leiden etwa $2 \%$ der Erwachsenen am Fibromyalgiesyndrom (FMS). Das Geschlechtsverhältnis ist zwar insgesamt ausgeglichen, jedoch dominieren unter den Patienten, die sich einer Behandlung unterziehen, Frauen: Über $80 \%$ der Patienten sind Frauen im Alter zwischen 40 und 60 Jahren.

2. Die von den Patientinnen mit FMS geschilderten Symptome haben sehr 


\begin{tabular}{l|ll} 
Tab. 1 & Basaler Hormonstatus der Patientin & \\
\hline Parameter & Ergebnis & Referenzbereich \\
\hline Estradiol & $74 \mathrm{pg} / \mathrm{ml}$ & $>30$ \\
\hline FSH & $11 \mathrm{U} / \mathrm{l}$ & $1,9-10$ \\
LH & $4 \mathrm{U} / \mathrm{l}$ & $1,0-9,0$ \\
TSH & $5,6 \mu \mathrm{U} / \mathrm{ml}$ & $0,27-4,2$ \\
fT3 & $3,1 \mathrm{pg} / \mathrm{ml}$ & $2,0-4,4$ \\
\hline fT4 & $12 \mathrm{ng} / \mathrm{l}$ & $9,3-17$ \\
\hline Kortisol & $103 \mathrm{ng} / \mathrm{ml}$ & $24,7-119$ \\
\hline Testosteron & $0,28 \mathrm{ng} / \mathrm{ml}$ & $0,08-0,48$ \\
Prolaktin & $21 \mathrm{ng} / \mathrm{ml}$ & $4,7-30,6$
\end{tabular}

häufig Bezug zu gynäkologischen

Krankheitsbildern oder endokrinen

Störungen, die in das Fachgebiet des

Frauenarztes gehören:

- Chronischer Unterbauchschmerz

- Dysmenorrhö

- Dyspareunie

- Vaginismus

- Vulvodynie

- Klimakterisches Syndrom

- Libidostörungen

- Antriebsstörungen

- Leistungsabfall

- Stimmungsschwankungen/ depressive Episoden

Dies stellt den Frauenarzt vor die Herausforderung abzugrenzen, welche Beschwerden einer klassisch somatisch ausgerichteten Behandlung zugänglich sind bzw. welche Symptome dem Fibromyalgiesyndrom zugeordnet werden müssen.

Aus den genannten Gründen sollen das Fibromyalgiesyndrom vorgestellt und die aktuellen Empfehlungen zur Diagnostik und Therapie dargestellt werden. Die Ausführungen basieren auf der aktuellen AWMF-Leitlinie [1].

\section{Das Fibromyalgiesyndrom}

\section{Definition und Klassifikation}

Das FMS ist durch folgende Kardinalsymptome charakterisiert:

- Chronische Schmerzen in mehreren Körperregionen

- Schlafstörungen

- Erschöpfungsneigung
Das FMS kann zwar mit depressiven Störungen assoziiert sein, ist jedoch nicht als solche zu klassifizieren. Es werden leichte, mittelschwere und schwere Verlaufsformen klassifiziert.

\section{Diagnostik}

Die Diagnose des FMS beruht auf der typischen Anamnese sowie dem Ausschluss somatischer Erkrankungen, welche die von der Patientin geschilderten Symptome hinreichend erklären. Zur Erfassung der genauen Schmerzlokalisation und -ausprägung werden Schmerzskizzen bzw. Schmerzskalen empfohlen. Ebenso sollte die Schlafstörung exakt beschrieben und dokumentiert werden sowie eine ausführliche Medikamentenanamnese erhoben werden.

Zum Ausschluss anderer Erkrankungen erfolgt die vollständige körperliche Untersuchung bzw. im Fall der gynäkologischen Patientin die entsprechende fachärztliche Untersuchung.

Zur Labordiagnostik werden folgende Parameter empfohlen [1]:

- BSG, CRP, kleines Blutbild (Ausschluss Polymyalgia rheumatica, rheumatoide Arthritis)

- Kreatinkinase (Ausschluss von Muskelerkrankungen)

- Kalzium (Ausschluss Hyperkalzämie)

- TSH, 25-OH-Vitamin D (Ausschluss Hypothyreose bzw. Vitamin-DMangel)

Je nach individueller Symptomatik muss darüber hinaus eine entsprechend spezifische Diagnostik veranlasst werden.

Ergeben sich bei der Exploration Hinweise auf seelische Symptombelas- tung bzw. anamnestische Hinweise auf psychiatrische Erkrankungen oder psychosoziale Stressoren sowie maladaptive Krankheitsverarbeitung, so sollte eine psychotherapeutische/psychiatrische Untersuchung veranlasst werden.

\section{Therapie}

Grundsätzlich lassen sich drei Säulen der Therapie beim FMS definieren:

1. Körperliche Aktivität; individuell angepasstes Ausdauer- und/oder Krafttraining

2. Medikamentöse Behandlung

3. Multimodale Therapie durch Kombination von mindestens einem aktivierenden Verfahren (Ausdauer-, Kraft- bzw. Flexibilitätstraining) mit mindestens einem psychotherapeutischen Verfahren (Patientenschulung und/oder kognitive Verhaltenstherapie)

Bei den leichten Formen des FMS wird der Patientin eine individuell angepasste körperliche Aktivität sowie die psychosoziale Aktivierung empfohlen (Pflegen von Hobbies sowie sozialen Kontakten). Bei schweren Verlaufsformen wird eine multimodale Therapie und ggf. eine zeitlich begrenzte medikamentöse Therapie empfohlen (s. unten).

\section{Ausdauertraining; Funktions- und Krafttraining}

Es wird empfohlen, Ausdauertraining mit geringer bis mittlerer Intensität (z. B. schnelles Spazierengehen, Walking, Fahrradfahren bzw. Ergometertraining, Tanzen, Aquajogging) 2- bis 3-mal/ Woche über mindestens $30 \mathrm{~min}$ zu praktizieren [2, 3]. Es scheint dabei keinen signifikanten Unterschied zwischen Wassergymnastik und Trockengymnastik zu geben. Sowohl Funktions- als auch Krafttraining mit jeweils 2- bis 3-mal/Woche für 30-60 min wird ebenso empfohlen.

\section{Medikamentöse Therapie}

Es gibt derzeit in Deutschland kein explizit für das FMS zugelassenes Medikament. 
Zur Behandlung des FMS können kurzfristig - insbesondere bei schweren Verläufen - folgende Substanzen eingesetzt werden:

$$
\begin{aligned}
& \text { - Amitriptylin (10-50 mg/Tag) } \\
& \text { - Duloxetin (60 mg/Tag) } \\
& \text { - Pregabalin (150-450 mg/Tag) }
\end{aligned}
$$

Starke Opioide sollen nicht gegeben werden, ebenso sollen keine nichtsteroidalen Antirheumatika verordnet werden.

Die Behandlung mit selektiven Serotoninwiederaufnahmehemmern (SSRI) kann allenfalls bei komorbiden depressiven und Angststörungen erwogen werden. Die Effekte auf die FMS-typischen Symptome sind sehr gering [4].

Für die Gabe von antiviralen Substanzen, Anxiolytika, Dopaminagonisten, Hormonen (Calcitonin, Testosteron, Östrogenen, Glukosteroiden, Schilddrüsenhormonen sowie Wachstumshormonen), Hypnotika, Interferonen sowie Lokalanästhetika wird in der aktuellen Leitlinie eine stark negative Empfehlung gegeben. Ebenso sind starke Opioide sowie Serotoninrezeptor(5- $\mathrm{HT}_{3}$ )-Antagonisten klar kontraindiziert [1].

Zusammenfassend ergibt sich daraus, dass keine wirksame medikamentöse Behandlung des FMS zur Verfügung steht. Auch wenn die Erwartungshaltung der Patientin oftmals dazu führt, dass ,gängige“ Schmerzmittel bis hin zu stark wirksamen Opiaten verordnet werden, so führt dies regelhaft nicht zu einer signifikanten Besserung der Beschwerden, mit hoher Wahrscheinlichkeit jedoch zu den typischen Nebenwirkungen.

\section{Multimodale Therapie}

Unter multimodaler Therapie des FMS versteht man die Kombination von mindestens einem aktivierenden Verfahren (Ausdauer-, Kraft- bzw. Flexibilitätstraining) mit mindestens einem psychotherapeutischen Verfahren (Patientenschulung und/oder kognitive Verhaltenstherapie). Es sollen mindestens drei der folgenden aktiven Therapieverfahren unter ärztlicher Leitung angewandt werden:

- Psychotherapie

- Spezielle Physiotherapie

- Entspannungsverfahren

- Ergotherapie

- Medizinische Trainingstherapie
- Sensomotorisches Training

- Arbeitsplatztraining, Kunst- oder Musiktherapie

Behandlungsverlauf und -erfolg der multimodalen Therapie werden systematisch überwacht und dokumentiert. Bei entsprechender Anwendung hat die multimodale Therapie einen guten Effekt auf den Schmerz und die Müdigkeit. Ebenso liess sich ein positiver Effekt auf die allgemeine Lebensqualität nachweisen. Von relevanten Nebenwirkungen ist bei dieser Therapie nicht auszugehen.

Daraus lässt sich die Schlussfolgerung ableiten, dass für die Langzeitbehandlung sowie für die Therapie schwerer Verlaufsformen die multimodale Therapie klar empfohlen werden kann und nachgewiesene Vorteile gegenüber der alleinigen Trainingstherapie bzw. der medikamentösen Behandlung aufweist [5-7].

\section{Fazit für die Praxis}

- Die Prävalenz des Fibromyalgiesyndroms (FMS) beträgt in Deutschland ca. $2 \%$ bei Erwachsenen. Etwa $80 \%$ der Patienten, die sich aufgrund des FMS behandeln lassen, sind Frauen im Alter zwischen 40 und 60 Jahren. Es gibt deutliche Überschneidungen zwischen den FMS-typischen Symptomen und gynäkologischen bzw. endokrinologischen Krankheitsbildern.

- Die Herausforderung für den Frauenarzt besteht darin abzugrenzen, ob es sich um somatisch begründete und entsprechend therapierbare Beschwerden handelt oder ob die Symptome dem Fibromyalgiesyndrom zuzuordnen sind.

- Um diese Abgrenzung sicher vornehmen zu können, sollte die Kooperation mit psychosomatischen Fachkollegen erfolgen, um bei Nachweis eines FMS für die betroffene Patientin die leitliniengerechte Therapie einzuleiten.

\section{Korrespondenzadresse}

Prof. Dr. med. Christoph Keck

amedes Holding GmbH

Haferweg 40, 22769 Hamburg, Deutschland

Christoph.Keck@amedes-group.com

\section{Einhaltung ethischer Richtlinien}

Interessenkonflikt. C. Keck gibt an, dass kein Interessenkonflikt besteht.

Für diesen Beitrag wurden vom Autor keine Studien an Menschen oder Tieren durchgeführt. Für die aufgeführten Studien gelten die jeweils dort angegebenen ethischen Richtlinien. Für Bildmaterial oder anderweitige Angaben innerhalb des Manuskripts, über die Patienten zu identifizieren sind, liegt von ihnen und/ oder ihren gesetzlichen Vertretern eine schriftliche Einwilligung vor.

Open Access. Dieser Artikel wird unter der Creative Commons Namensnennung 4.0 International Lizenz veröffentlicht, welche die Nutzung, Vervielfältigung, Bearbeitung, Verbreitung und Wiedergabe in jeglichem Medium und Format erlaubt, sofern Sie den/die ursprünglichen Autor(en) und die Quelle ordnungsgemäß nennen, einen Link zur Creative Commons Lizenz beifügen und angeben, ob Änderungen vorgenommen wurden.

Die in diesem Artikel enthaltenen Bilder und sonstiges Drittmaterial unterliegen ebenfalls der genannten Creative Commons Lizenz, sofern sich aus der Abbildungslegende nichts anderes ergibt. Sofern das betreffende Material nicht unter der genannten Creative Commons Lizenz steht und die betreffende Handlung nicht nach gesetzlichen Vorschriften erlaubt ist, ist für die oben aufgeführten Weiterverwendungen des Materials die Einwilligung des jeweiligen Rechteinhabers einzuholen.

Weitere Details zur Lizenz entnehmen Sie bitte der Lizenzinformation auf http://creativecommons.org/ licenses/by/4.0/deed.de.

\section{Literatur}

1. AWMF (2017) Definition, Pathophysiologie, Diagnostik und Therapie des Fibromyalgiesyndroms. 2. Aktualisierung. http:www.awmf.org/leitlinien/ detail/LL/145-004.html. Zugegriffen:02.05.2020

2. Bidonde J, Busch AJ, Webber SC, Schachter $\mathrm{CL}$, Danyliw A, Overend TJ et al (2014) Aquatic exercise training for fibromyalgia. Cochrane Database Syst Rev 10:CD11336. https://doi.org/10.1002/ 14651858.CD011336

3. Busch AJ, Webber SC, Richards RS, Bidonde J, Schachter CL, Schafer LA et al (2013) Resistance exercise training for fibromyalgia. Cochrane DatabaseSyst Rev 12:CD10884. https://doi.org/10. 1002/14651858.CD010884

4. Walitt B, Urrútia G, Nishishinya MB, Cantrell $S E$, Häuser W (2015) Selective serotonin reuptake inhibitors for fibromyalgia syndrome. Cochrane Database Syst Rev 6:CD11735. https://doi.org/10. 1002/14651858.CD011735

5. Häuser W, Bernardy K, Arnold B, Offenbächer M, Schiltenwolf M (2009) Efficacy of multicomponent 
Fachnachrichten

treatment in fibromyalgia syndrome: a metaanalysis of randomized controlled clinical trials. Arthritis Rheum 61(2):216-224. https://doi.org/ 10.1002/art.24276

6. Petzke F, Brückle W, Eidmann U, Heldmann P, Köllner V, Kühn T et al (2017) Aktualisierte Leitlinie und systematische Übersicht von systematischen Übersichtsarbeiten: Allgemeine Behandlungsgrundsätze, Versorgungskoordination und Patientenschulung beim Fibromyalgiesyndrom. Schmerz 31:246-254. https://doi.org/10.1007/ s00482-017-0201-6

7. de Rooij A, Roorda LD, Otten RHJ, van der Leeden M, Dekker J, Steultjens MPM (2013)

Predictors of multidisciplinary treatment outcome in fibromyalgia: a systematic review. Disabil Rehabil 35:437-449. https://doi.org/10.3109/ 09638288.2012.699582

Hinweis des Verlags. Der Verlag bleibt in Hinblick auf geografische Zuordnungen und Gebietsbezeichnungen in veröffentlichten Karten und Institutsadressen neutral.

\section{Deutsche Menopause Gesellschaft e.V.}

Ausschreibungen 2020

\section{NEUES AUS DER WISSENSCHAFT}

Die Deutsche Menopause Gesellschaft e.V. (DMG) verleiht drei Preise an junge wie auch erfahrene Wissenschaftler, die auf dem Gebiet der Peri- und Postmenopause und angrenzenden Bereichen wie Diabetologie, Ernährungswissenschaft, Innere Medizin, Kardiologie, Onkologie, Ophthalmologie , Osteologie, Neurologie, Psychiatrie, Psychologie, Epidemiologie, etc. forschen.

Diesen Vorträgen wird am 13.11.2020 während der Jahrestagung der DMG e.V. ein ganzer Vormittag gewidmet.

Die drei besten wissenschaftlichen Vorträge werden mit 1.250 Euro, 750 Euro und 500 Euro ausgezeichnet.

Alle, die zu einem Vortrag eingeladen werden

- entrichten keine Teilnehmergebühr

- können das gesamte wissenschaftliche Programm der Tagung besuchen

- erhalten eine Unterstützung der Reisekosten und

- die kostenfreie Mitgliedschaft in der DMG e.V. für ein Jahr sowie

- ein Abonnement inkl. Online-Zugang für die Zeitschrift Gynäkologische Endokrinologie

Einreichungen können aus Deutschland, Österreich und der Schweiz in deutscher oder englischer Sprache erfolgen.

Vorgaben zur Form siehe "Hinweise zur Abstracteinreichung" unter www.wissenschaftspreis-dmg.de oder www.menopause-gesellschaft.de

Deadline für die Einreichung Ihres Abstracts ist der 31. August 2020 an <softconsult@web.de> Betreff "Neue Wissenschaft"

\section{CHRISTIAN-LAURITZEN-PREIS}

Die Deutsche Menopause Gesellschaft e.V. stellt aus Mitteln des Förderkreises dieser Gesellschaft zur Prämierung hervorragender wissenschaftlicher Originalarbeiten jährlich einen Gesamtbetrag von 5.000 Euro zur Verfügung.

Preiswürdige Arbeiten müssen neue Ergebnisse klinischer Forschung darstellen, die sich primär mit der sexualhormonabhängigen Gesundheit von Frauen und Männern in der zweiten Lebenshälfte auseinandersetzt. Eingeschlossen sind auch experimentelle Arbeiten, die eine unmittelbare Beziehung zu dieser Fragestellung herstellen.

\section{Einzureichende Unterlagen}

Ein aussagekräftiges Manuskript, max. 30 Seiten (DIN-A 4, computergestützte Texterstellung, inklusive Abbildungen, Tabellen und Referenzen; deutsch oder englisch), das den Autorenrichtlinien einer im Jahr der Einreichung im Index Medicus gelisteten Zeitschrift entspricht. Es ist als "PDF" per Email sowie 1x in Papierform an die Geschäftsstelle zu schicken.

Weitere Informationen zur Einreichung sowie die Satzung des Christian-LauritzenPreises finden Sie unter www.menopausegesellschaft.de

\section{Bewerbungsfrist ist der} 31. August 2020

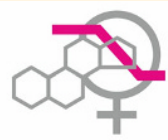

Deutsche Menopause Gesellschaft e.V. Präsidentin: Dr. Katrin Schaudig

Geschäftsstelle: Anne Becker Weißdornweg 17 - 35041 Marburg

+49 (0) 6420 - 93444

E-mail:Info-DMG@email.de www.menopause-gesellschaft.de 